\title{
A Nationwide Study on Optical Analysis for Expecting HEOs to Support Ambulances
}

\author{
Isao Nakajima ${ }^{1 *}$, Kazuhide Tsuda $^{2}$, Hiroshi Juzoji ${ }^{3}$, Masuhisa Ta ${ }^{4}$, Atsushi Nakajima ${ }^{5}$
}

\begin{abstract}
This paper deals with actual optical data from rural as well as urban areas in a nationwide study captured with Fisheye cameras. Simultaneously data was collected (of the receiving power density) from the mobile communications satellite N-STAR. The visibility of the satellite is easily determined by checking the value of the pixels in the binarized fisheye image of its position. The process of determining the visible satellite is automatically performed. Based on the analyses of the field data measured in Japan, we are expecting HEOs (Highly inclined Elliptical Orbiters) that would reduce blockage in the extreme northern region of Wakkanai City well as in the most crowded urban area, in Tokyo Ginza. In case of HEOs operation, the elevation angle will improve from 37 with N-STAR GEO to 75 degrees.

HEOs could replace 5G/Ka-band or support in rural areas where broadband circuit is not available. We are proposing combination operations with HEOs and 5G/Ka-band to solve blockage problems, because HEOs can keep line-of-sight propagation with high elevation angle for long duration. In such operations, the communications profile on the vehicle based on actual optical data will be very useful to predict blockages and to select/switch a suitable circuit.
\end{abstract}

Key Words: 5G, fisheye camera, mobile communication profile

\section{INTRODUCTION}

The purpose of this paper is to promote mobile and ambulatory communications, especially for ambulances. This paper discusses optical data communication in urban and suburban areas of Japan via fisheye cameras. The approach proposed would permit the recording of simultaneously captured digital data received via the Sband from a mobile communications satellite (NTT DoCoMo's N-STAR GEO) with an onboard tracking system, based on directional and positioning data obtained by a GPS gyrocompass.

This paper proposes methods, experiments, and a model for obtaining a nationwide communication profile to help predict the propagation of mobile communication satellites and to support ambulatory applications, including forward video transmission links. We emphasize the benefits of deploying HEOs (Figure 1) to address issues associated with obstructions to line-of-sight communications.

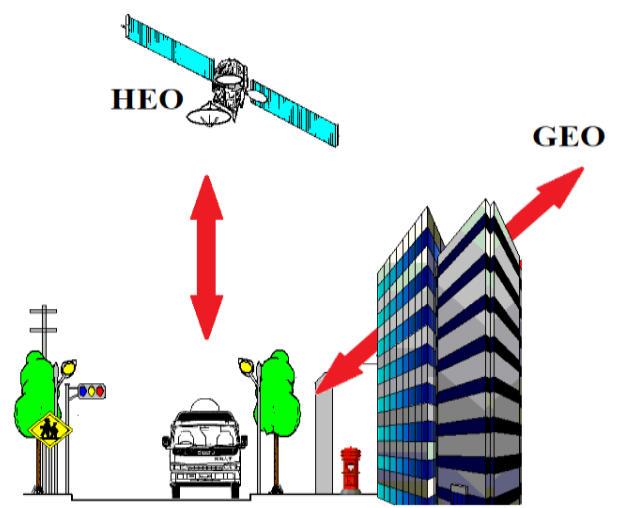

Fig. 1. Highly inclined elliptical Orbiter (HEO) to send a real-time video from ambulance.

\section{BACKGROUND}

\subsection{Forward linkage of video transmission from moving vehicles.}

Propagation effects with the LMSS (Land Mobile Satellite Service) differ from those for fixed satellite service

\footnotetext{
Manuscript received June 05, 2019; Revised June 18; Accepted June 19, 2019. (ID No. JMIS-19M-06-021)

Corresponding Author (*): Isao Nakajima, Shimokasuya 143, Isehara City, Japan, +81-463-91-3130, js2hb@ets8.jp

${ }^{1}$ Nakajima Lab. Tokai University School of Medicine, Isehara City, Japan, js2hb@ets8.jp,

${ }^{2}$ Nakajima Lab. Tokai University School of Medicine, Isehara City, Japan, umanari.com@gmail.com

${ }^{3}$ EFL Inc. Takaoka City, Japan, juzoji@yahoo.co.jp.

${ }^{4}$ Tasada Works Ltd., Takaoka City, Japan, tasada@lilac.ocn.ne.jp

${ }^{5}$ Nakajima Space Engineering Lab., Chofu City, Japan, nalnakajima@hotmail.com
} 
(FSS), primarily due to the greater significance of terrain effects, including trees, buildings, hills, and mountains.

FSS generally allows discrimination against multipathing, shadowing, and obstructions through the use of highly directional line-of-sight antennas placed at unobstructed sites.

The third-generation (3G) mobile communication technologies already introduced in developed nations address certain data transmissions (64 kbps $\mathrm{x} \mathrm{n}$ ) from moving vehicles in urban areas. However, precise observations of patient conditions by clinical physicians depend on high-quality images of $2 \mathrm{Mbps}$ and/or $10 \mathrm{Mbps}$, with compressed HDTV from ambulances en route. Certain clinical cases require high-quality images to assess the condition of patients in critical condition, such as HDTV compressed with HEVC (High Efficiency Video Coding, or H.265), transmitted from moving ambulances.

\subsection{The 3G cellular phone}

The third-generation wireless cellular phone (the 3G wireless mobile phone) is called the $3 \mathrm{G}$ phase 1 , or Wideband CDMA in Japan. NTT DoCoMo has already offered full service in $99 \%$ of the surface area of Japan. In the transmission of video data from an ambulance to an emergency center, the transmission capability of the upstream line (from the mobile station to the transit node) is of great importance. NTT DoCoMo offers three kinds of upstream line services. As ambulances run at a maximum $80 \mathrm{~km} / \mathrm{h}$ on the street, the data transmission is to be conducted at a rate of $144 \mathrm{kbps}$ by FDD (Frequency Division Duplex) via macro Node B over a maximum transmission distance of $15 \mathrm{~km}$. It is difficult to conduct upstream transmission at $2 \mathrm{Mbps}$ from a high speed vehicle in an environment in which-frequency selective fading will be generated due to multipath interference urban areas.

\subsection{G/Ka-band}

$5 \mathrm{G} / \mathrm{Ka}$-band is expected to support significantly faster mobile broadband speeds and higher volumes of data use than previous generations (the latter is based on the LTE, or Long-Term Evolution, standard). The ITU-R standard for wireless broadband communication will expand the functionality of mobile devices and data terminals while unleashing the full potential of the Internet of Things. As a core fiber-through-the-air technology, $5 \mathrm{G}$ is expected to support autonomous cars in smart cities and the industrial Internet and play a key role in the future of telecommunications. $5 \mathrm{G} / \mathrm{Ka}$-band will also prove essential in maintaining today's most popular mobile applications, including on-demand video, by ensuring that growing uptake and usage can be sustained.

However, 5G/Ka-band uses wideband frequency resources with $500 \mathrm{MHz}$ bandwidth on $28 \mathrm{GHz}$. This means the frequency characteristics related to blockage and shadowing are dramatically different from the lower microwave frequencies. Communication distances for $5 \mathrm{G} / \mathrm{Ka}$-band will be $100-200 \mathrm{~m}$. Since the operating principle is primarily line-of-sight, carriers will need to deploy many repeaters and transponders across the urban landscape.

Since HEO can cover Ka-band obstructions, we expect HEO to prove highly effective in supporting forward video transmission from moving vehicles to emergency triage centers.

\section{METHODS}

\subsection{Mathematical approach to fading problem}

Multipath fading is due to the constructive and destructive combination of randomly delayed, reflected, scattered, and diffracted signal components. Under the multipath environment, there are three models designed mathematically.

\subsubsection{Nakagami-Ricean fading}

The Nakagami-Ricean density distribution is given by formula (1)

$$
p(x)=\frac{2 x}{M_{\mathrm{r}, \mathrm{A}}} \exp \left(-\frac{1+x^{2}}{M_{\mathrm{r}, \mathrm{A}}}\right) I_{0}\left(\frac{2 x}{M_{\mathrm{r}, \mathrm{A}}}\right)
$$

Io : Zero-order Bessel function

$\mathrm{M}_{\mathrm{rA}}$ : average power of scattered wave component

$K$ : Rice coefficient

$$
\begin{aligned}
& 2 \sigma^{2}=M_{\mathrm{r}, \mathrm{A}} \\
& K=\frac{1}{M_{\mathrm{r}, \mathrm{A}}}
\end{aligned}
$$

This propagation environment consists of one strong direct wave (line-of-sight) and weak multiplex propagation waves.

\subsubsection{Loo distribution}

It is assumed that the received signal is affected by nonselective Rice fading with lognormal shadowing on the direct component only, while the diffuse scattered component has constant average power level. It means that a direct wave declines by lognormal distribution caused by leaves of roadside trees. In such propagation environment, a synthetic wave ingredient will serve as Loo distribution.

$$
\begin{gathered}
p(x)=\frac{6.930 x}{\sigma M_{\mathrm{r}, \mathrm{B}}} \int_{0}^{\infty} \frac{1}{z} \exp \left[-\frac{[20 \log z-m]^{2}}{2 \sigma^{2}}-\frac{x^{2}+z^{2}}{M_{\mathrm{r}, \mathrm{B}}}\right] I_{0}\left(\frac{2 x z}{M_{\mathrm{r}, \mathrm{B}}}\right) \mathrm{d} z \\
p(x)=\frac{2 x}{M_{\mathrm{r}, \mathrm{C}}} \exp \left(-\frac{x^{2}}{M_{\mathrm{r}, \mathrm{C}}}\right)
\end{gathered}
$$

$\mathrm{M}_{\mathrm{rB}}$ : average power of scattered wave component 3.1.3. Rayleigh distribution

When a line-of-sight signals are influenced by buildings, the direct path is entirely blocked. This propagation 
condition is referred as deep shadowing state, a synthetic signal is based on stray multipath components which obey Rayleigh distribution.

$$
p(x)=\frac{2 x}{M_{\mathrm{r}, \mathrm{C}}} \exp \left(-\frac{x^{2}}{M_{\mathrm{r}, \mathrm{C}}}\right)
$$

$\mathrm{M}_{\mathrm{rC}}$ : average power of scattered wave component

$$
P\left(x_{0}\right)=1-\exp \left(-\frac{x_{0}^{2}}{M_{\mathrm{r}, \mathrm{C}}}\right)
$$

\subsection{Markov chain model}

Fading among channel states is a relatively slow process if the vehicle is relatively distant from buildings or trees (for example, 5 to $10 \mathrm{~m}$ ). This fading is determined by the distance of user movement and elevation change, each state maintaining a certain geometric space. The change in states is described by the Markov chain process. When a mobile terminal moves over a certain distance, it switches randomly to a new state. Figure 2 illustrates modeling for the three-state Markov process. We observe a strong relationship between the three propagations of the fading channel and visibility (optical path) taken by fisheye camera shown in Table 1.
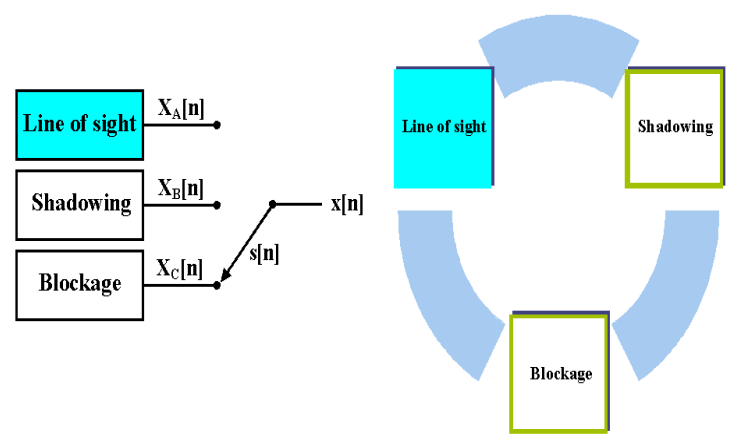

Fig. 2. Markov chain of the propagation.

Table 1. Schema of visibility and microwave propagation

\begin{tabular}{|l|l|}
\hline Microwave propagation & \multicolumn{1}{|l|}{ Optics } \\
\hline Line-of-sight & $\begin{array}{l}\text { Ricean } \\
\text { fading }\end{array}$ \\
\hline Shadowing & $\begin{array}{l}\text { Loo } \\
\text { distribution }\end{array}$ \\
\hline Blockage & $\begin{array}{l}\text { Rayleigh } \\
\text { fading }\end{array}$ \\
\hline
\end{tabular}

\subsection{Analysis of the optical method}

\subsubsection{How to obtain optical data}

We captured the images recorded by the video system using a computer, and converted them into digital motion pictures. The motion-picture files (in the AVI format) were converted to still images corresponding to each frame (bit map). To enable the easy analysis of images, the color images were converted to monochrome binary (two-level) images (Figure 3) in accordance with a predetermined threshold.

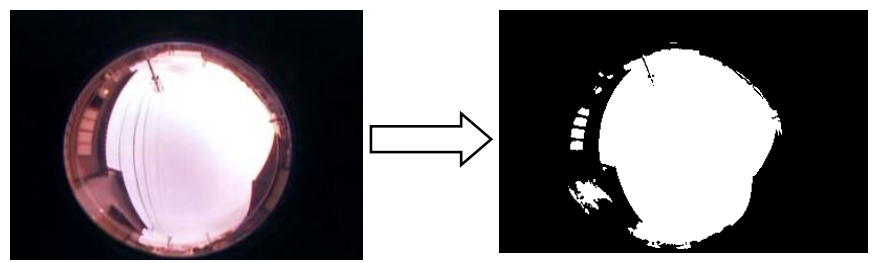

Fig. 3. Conversion to binary images.

The length from the center to the first black region of the binary image gives the minimum elevation angle in the corresponding direction. On the other hand, direction from the center to the bottom of the image agrees with the running direction. Thus, if this direction is matched with the direction indicated by the GPS compass (the running direction of the vehicle indicated by the data synchronized using the time code and the GPS timer), the running direction of the vehicle can be calculated as an absolute value.

\subsubsection{Coordinate conversion model of El and $A z$}

First, we can calculate the satellite position from the (x,y) coordinates of the two-dimensional binary images. To transform the $(\mathrm{El}, \mathrm{Az})$ coordinates from the $(\mathrm{x}, \mathrm{y})$ coordinates, we have to model geometric correlation shown in the Figure 4. With the following equations, we can calculate the elevation and the azimuth of the target satellite.

$\mathrm{x}, \mathrm{y}$ : coordinates of the two-dimensional still image

El, Az: gimbal coordinates of the target satellite

$\mathrm{U}, \mathrm{V}$ : tangent plane at the target point of the lens

$\mathrm{R}$ : radius of the lens

f: focal length

star mark: target satellite

$\theta$ : angle of the tangent plane and $\mathrm{x}$-axis

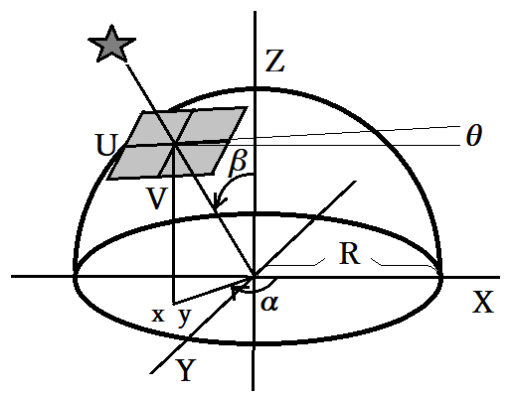

Fig. 4. The coordinate conversion model of $\mathrm{El}$ and $\mathrm{Az}$ using a fisheye lens. 


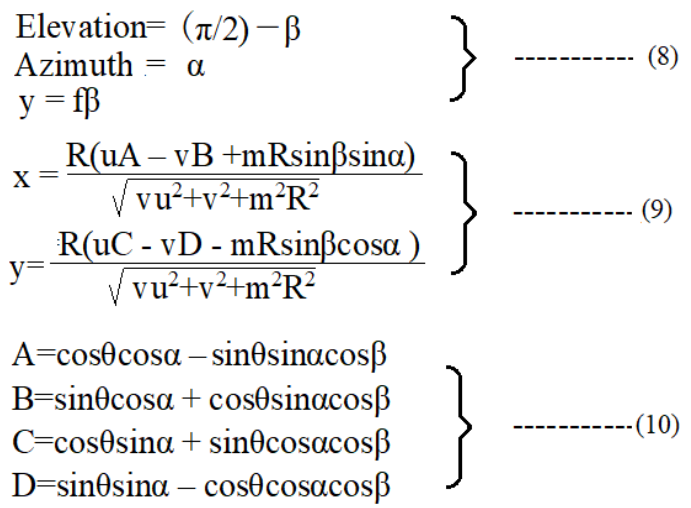

\subsection{Hardware}

Described above is the concept. Actual implementation of the scheme requires mounting the satellite receiver so as to obtain the beacon signal from N-STAR; deploying a fisheye camera with graphic image recorders; using multichannel analog records for the linear data from the experimental vehicle; leveraging GPS navigation data; and using the direction of vehicle movement detected by the GPS gyro (Figure 5, 6, 7).

\subsubsection{Fisheye camera system}

We installed the fisheye camera system on the top of the roof of the vehicle. Since the lens (Nikon FC-E8, circular image, focal length of $8 \mathrm{~mm}$ covering 183 degree) is designed only for COOLPIX video camera, so we have to convert image with $\mathrm{C}$ mount adapter. It recorded with the HDTV video recorders (Panasonic DVCPRO 50, 30 flams $/ \mathrm{sec}$.) Also, we mounted HDTV video camera to take a front view of the vehicle.

\subsubsection{The N-STAR Mark-a tracking system}

The N-STAR Mark-a satellites will provide fixed and mobile telecommunications services for a design life of over 10 years over the Pacific Ocean. N-STAR Mark-a provided telecommunications coverage for the Japanese Islands and its territorial waters. We used this beacon signal on $2.5010 \mathrm{MHz}$ as a pilot signal, designed a 7.5 tern helical antenna $(12 \mathrm{dBi}, \mathrm{C}=0.95$; axial type) on $\mathrm{S}$-band with tracking motor (max. angler speed $30 \mathrm{deg} . / \mathrm{sec}$.) based on the vehicle's direction data obtained by GPS gyro. The $\mathrm{C} / \mathrm{N}$ of the beacon signal was over $10 \mathrm{~dB}$ at the line of sight propagation. However, in case of severe multipath environment at an urban area, we selected the phase composition with 4 helical antennas to detect fine direction of the satellite.

\subsubsection{GPS satellite compass}

We installed a high-precision Furuno GPS satellite compass (SC-60, accurate $+/-0.6$ deg.) on the roof of the vehicle to ensure super heading accuracy. This compass is capable of identifying rapid turns of up to $45 \mathrm{deg}$./sec. The unique tri-antenna system improves accuracy and reduces the effects of yaw, pitch, and roll.

Since this system outputs information only on the vehicle's heading, identifying navigation information requires a separate commercial GPS receiver.

\subsubsection{Driving data collection}

We integrated multiple channels data to the hard disk of the PC, such as the optical 3 axis gyroscope (FOG: Fiber Optical Gyro by Japan Aviation Electronic Industry), 3 axis acceleration, the pulse (velocity pulse) from a wheel, steering angle, data of a accelerator, and data of a brake. The sampling rate of the A/D converter for the FOG was $15 / \mathrm{sec}$. with 12 bits. This speed is based on the characteristic of FOG for public application recommended by the COCOM (Coordinating Committee for Multilateral Export Controls) and is not the limit speed of an A/D conversion.

\subsubsection{Integrated image for the space-time management}

The position data, date and time, heading, and speed, provided by the GPS satellite compass were superimposed on the images of the front and zenith views captured by the fisheye camera.

The vehicle heading, speed, acceleration, navigation data, and date/time were recorded on the hard disk of the vehicle orientation measurement system. The time code for the digital video camera was matched to GPS time, and the video camera was synchronized with the vehicle orientation measurement system with reference to GPS time. The two video systems mutually synchronized based on the common time code.

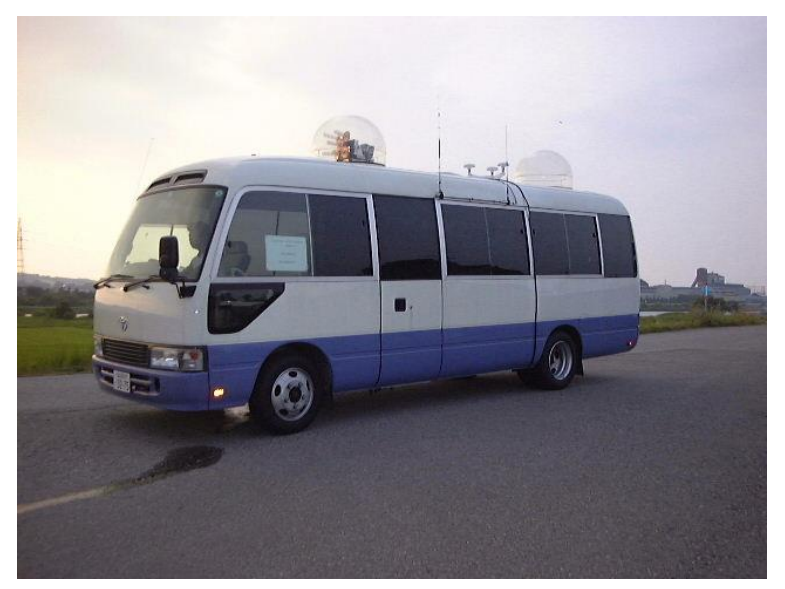

Fig. 5. The experiment vehicle. 


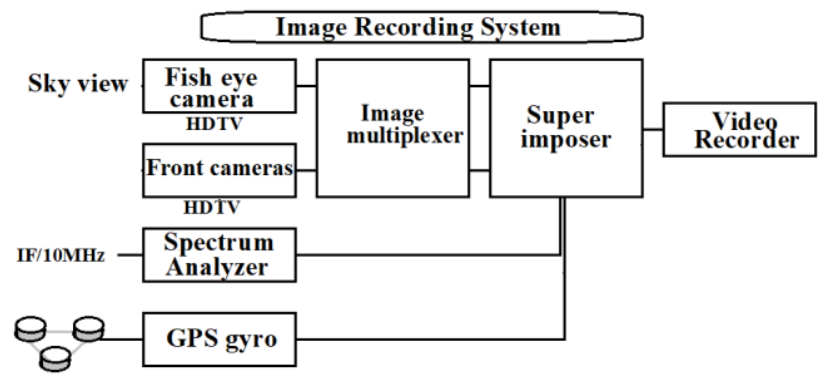

Fig. 6. Configuration of image recording

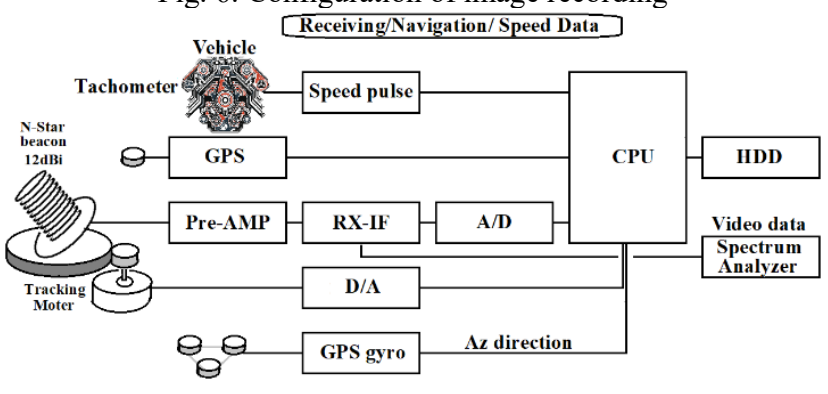

Fig. 7. Vehicle driving data recorder.

\subsection{Field data collection}

We gathered field data based on travel over a total dista nce of approximately 10,000 km (Table 2, Figure 8).

In Table 2, the distances are acquired from the tachometer of the vehicle, also showed the elevation angle to N-STAR, average value of visibility to the South. It is very difficult to find out the optical feature from this average value to the South in each city. In common sense judgment, if location was north, a value will be low, if road width was wide, a value will be high. Then, investigation and analysis were conducted in detail to reflect from the spatial city feature more, such as the northernmost town or most crowded shopping area.

\subsection{Results of filed data}

Here we discuss the basic field data analysis in items A, $\mathrm{B}$, and C; the characteristics of Japan's northernmost city in item D; and the largest urban area in item E.

\section{A. Angle of elevation and driving direction}

Figure 9 shows the minimum elevation angles observed on the south side (180 degrees) during an east-to-west drive in the suburbs. In contrast, a drive in the 45-degree direction, i.e., northeast, showed virtually no obstacles. The transverse axis represents the number of frames (distance $=$ velocity $\times$ time). The obstacles that periodically interfere with optical visibility are utility poles.

B. Multipath and Loo distribution

In order to confirm the Loo distribution and multipath effect on the S-band, we drove the experiment vehicle slowly along a road near a building and recorded the angle of elevation and the voltage of the received signal from the satellite (Figure 10).
Table 2. Operation distance nation-wide.

\begin{tabular}{|l|c|c|c|}
\hline Area & Distance[km] & $\begin{array}{c}\text { Elevation a } \\
\text { ngle to N- } \\
\text { STAR[deg.] }\end{array}$ & $\begin{array}{c}\text { Average } \\
\text { of visib } \\
\text { ility to } \\
\text { the Sou } \\
\text { th[\%] }\end{array}$ \\
\hline Wakkanai & 64 & 36.8 & 85.5 \\
\hline Nemuro & 43 & 38.2 & 85.0 \\
\hline Sapporo & 72 & 39.4 & 82.5 \\
\hline Sendai & 100 & 44.7 & 77.0 \\
\hline Tokyo & 564 & 47.8 & 84.0 \\
\hline Yokohama & 94 & 48.1 & 85.5 \\
\hline Nagoya & 48 & 48.8 & 88.0 \\
\hline Osaka & 64 & 49.5 & 87.5 \\
\hline Okayama & 29 & 49.7 & 89.5 \\
\hline Hiroshima & 80 & 50.0 & 91.0 \\
\hline Tottori & 13 & 48.7 & 89.0 \\
\hline Takamatsu & 17 & 50.1 & 80.0 \\
\hline Kochi & 20 & 50.9 & 85.5 \\
\hline Fukuoka & 59 & 50.9 & 87.0 \\
\hline Naha & 32 & 59.0 & 93.0 \\
\hline Hokkaido round & 3100 & & \\
\hline Kyusyu round & 991 & & \\
\hline Shuto Expressway & 14 & & \\
\hline Tokyo-Aomori & 1360 & & \\
\hline Isehara-Fukuoka & 2810 & & \\
\hline Tokyo-Nagoya & 690 & & \\
\hline Tokyo-Niigata & 410 & & \\
\hline Takamatsu-Kochi & 248 & & \\
\hline Okinawa & 114 & & \\
\hline Other areas & 96 & & \\
\hline
\end{tabular}

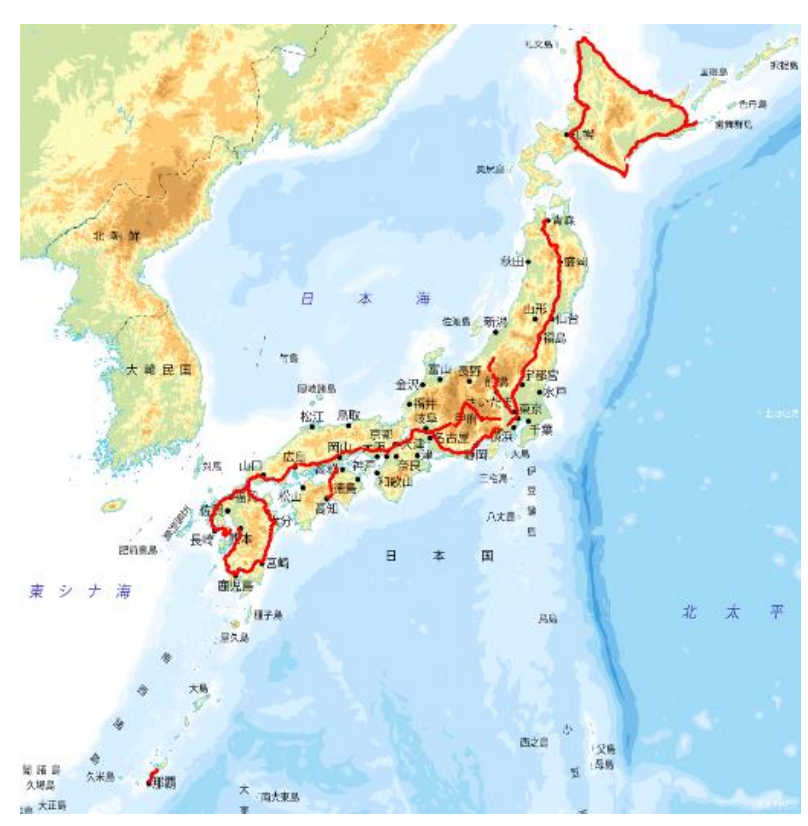

Fig. 8. Collected field data nation wide. 

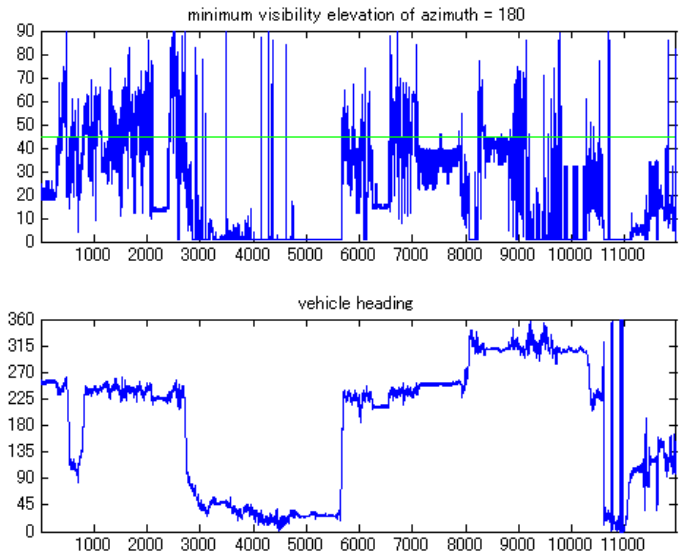

Fig. 9. Minimum elevation angles for obstacles observed in a downtown area during a drive from east to west. Note the numerous obstacles to the south as Japanese traffic environment.

We encountered two types of time lags, A and B, between the elevation angle and the voltage of the received signal._It is reasonable to suspect reflective waves_(multipathing) will affect A more than B, and that this multipathing is due to differences in geometric structure from building to building and/or the direction to the satellite. We accept that errors will result when applying optical measurements to microwaves at low frequencies like the S-band. These errors will shrink with higher frequencies.

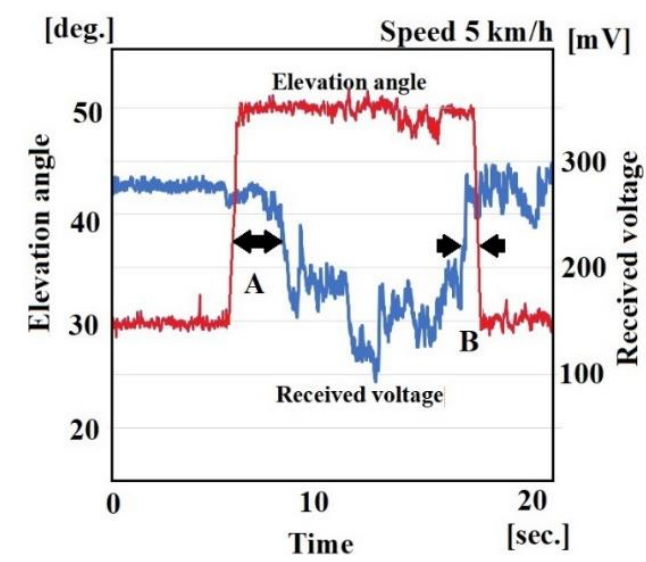

Fig. 10. Relationship between received voltage and blockage, time lag A: going into the blockage, time lag B: going out from the blockage of the building.

\section{Distance of blockage}

Meanwhile, obstacles appear continuously 30-40 degrees during the drive in the downtown area. These are buildings (single-family homes in the traditional Japanese style). We plotted the data obtained to show the cumulative frequency of obstacle lengths in Figure 11 for the downtown area.

The distance of the blockage becomes $2.3 \mathrm{~m}$ at an angle of elevation of 60 degrees. The most common obstacles blocking optical line-of-sight are utility poles of less than 5 $\mathrm{m}$ in height. Both in the suburbs and downtown, all obstacles other than pedestrian bridges were less than $2 \mathrm{~m}$ in height. Thus, the spatial diversity (width of the base between the two antennas ) on the vehicle must be at least $233 \mathrm{~cm}$.

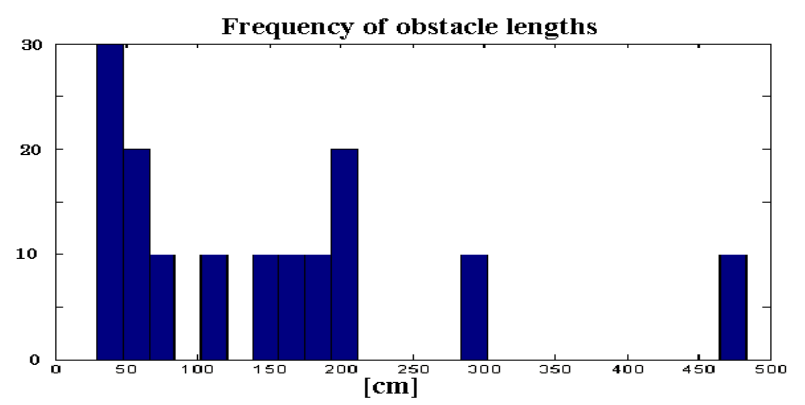

Fig. 11. Cumulative frequency of obstacle lengths in the downtown area.

\section{The extreme northern region of Wakkanai City}

Wakkanai lies in the extreme north of Japan, located just $40 \mathrm{~km}$ from the island of Sakhalin. The angle of elevation to N-STAR Mark-a is just 36.8 degree. Thus, we investigated the angle of elevation and blockages. Figure 12 shows the route in Hokkaido, including Wakkanai.

With Japanese road environments in mind, we analyzed the relationship between visibility and azimuth angle to the south. We drove the vehicle on the test courses in downtown Wakkanai, including those extending in different directions at 45 -degree intervals to 360 degrees. Here, we calculated the cumulative angle of elevation and visibility as percentage values based on a test drive over a relatively narrow road in downtown Wakkanai. Since cars proceed on the left in Japan and approaching buildings therefore lie to the left, Figure 13 can be interpreted to indicate the significant obstruction to the south caused by built structures.

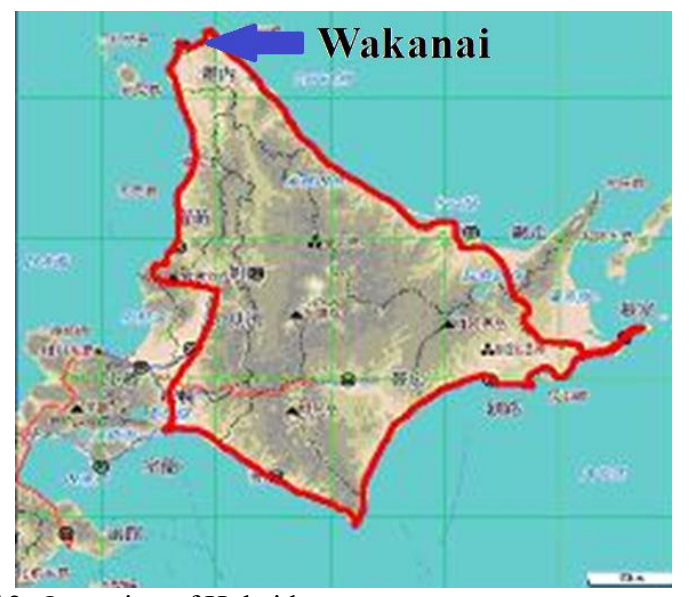

Fig. 12. Operation of Hokaido. 


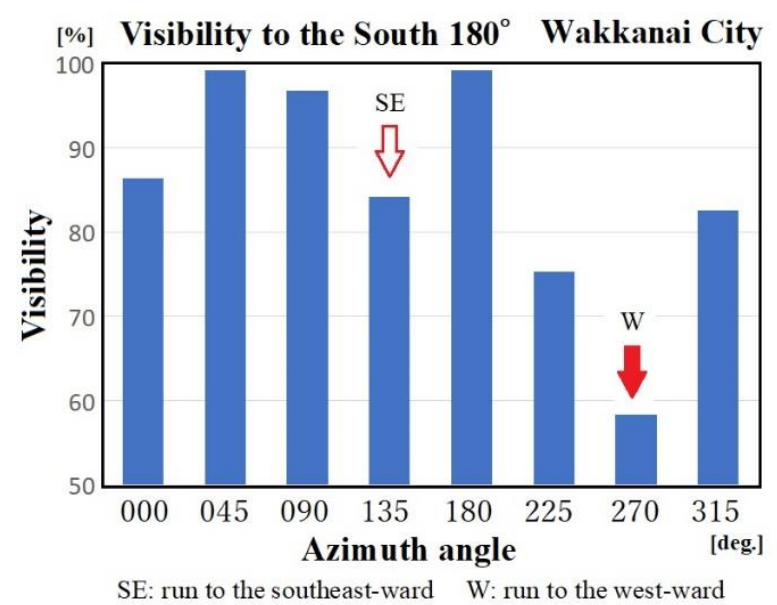

Fig. 13. The relationship between visibility and azimuth angle to the South, Wakkanai City

\section{E. Tokyo urban area: Ginza}

Ginza is currently regarded as the most densely built area in Japan. The angle of elevation to N-STAR Mark-a is 48.2 degrees. Ginza is characterized by numerous narrow alleys connected to main streets (in red or blue; Figure 14)

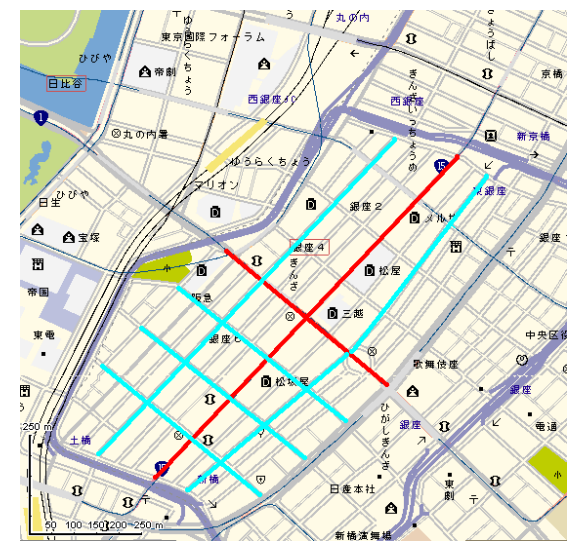

Fig. 14. Map of Ginza, densely urbanized Tokyo neighborhood characterized by many narrow alleys connected to main streets

Here, we report on an analysis of visibility to the south (azimuth 180 degrees). Figure 15 gives overall averages, Figure 16 data for alleys, and Figure 17 data for main streets. In each figure, black represents actual field data, dark gray is urban, and light gray is suburban simulated by Prof. Yoshio Karasawa's Formula (11) published in IEEE Trans. VT[06] .

Visibility $=1-\mathrm{a}(90-\mathrm{El})^{2}$

El: elevation angle[degree] $>10 \mathrm{deg}$.

a : $1.43 \times 10^{-4}:$ urban

a: $6.0 \times 10^{-5}:$ suburban
Based on this result, the curve from 20 to 50 degrees is almost the same as Karasawa's expectation, and the alleys in Ginza have severe blockages from 10 to 50 degrees. For these reasons, we anticipate HEOs will improve performance near these obstructions and enhance 5G communication for moving platforms in Japan's urban centers.

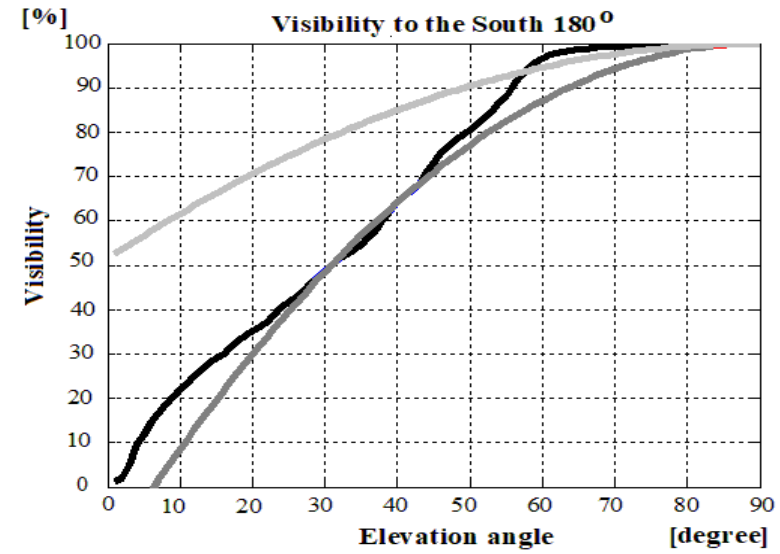

Fig. 15. The average of the alleyway and main street.

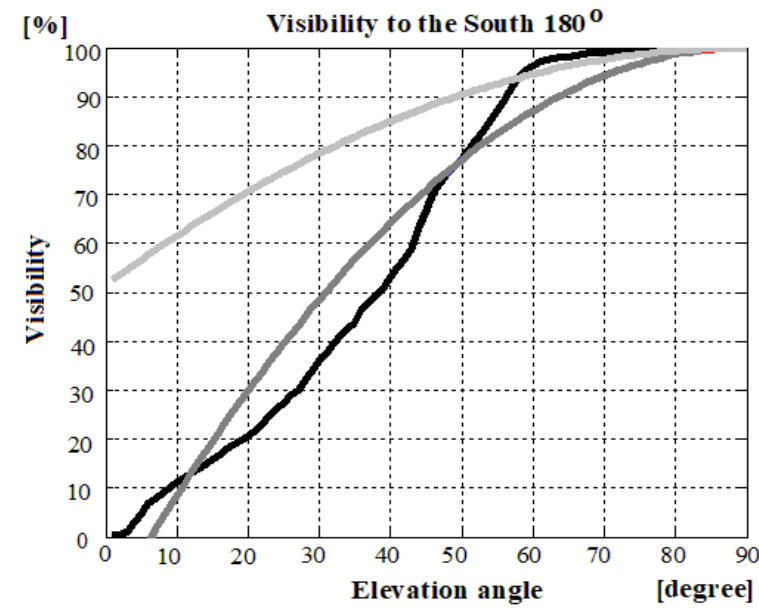

Fig. 16. Alleyway in Ginza.

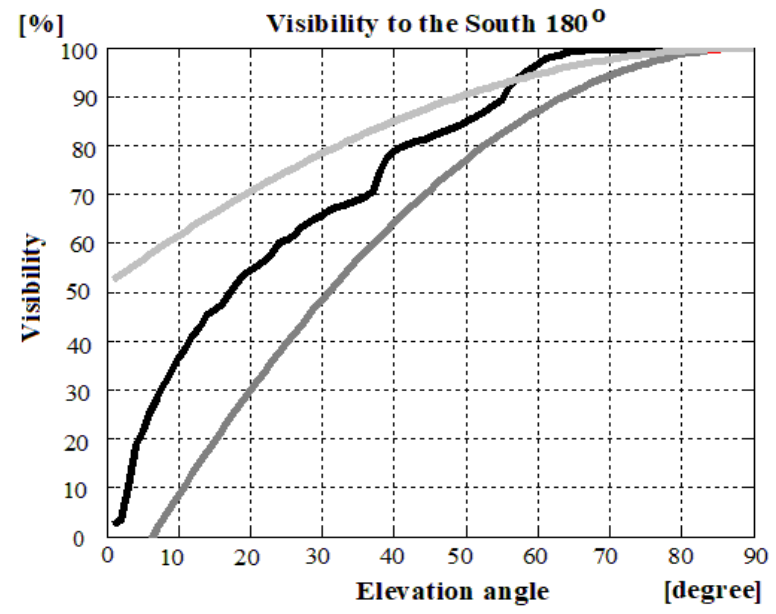

Fig. 17. Main street in Ginza. 


\section{CONSIDERRATIONS}

\subsection{Distance of the diversity antenna}

Optical measurement can be a highly effective way to detect obstacles in urban environments, assuming the method presented here is applied along with a method for recognizing differences based on the blockage of microwave propagation. All obstacles other than overpasses and tunnels do not exceed $2.3 \mathrm{~m}$ in distance.

Thus, we determined the optimal spatial diversity for ambulatory applications to be $2.3 \mathrm{~m}$. Prof. Kitano has pointed out the benefits of using a diversity antenna to track two satellites (GEO) published IEEE Trans. AES [07].

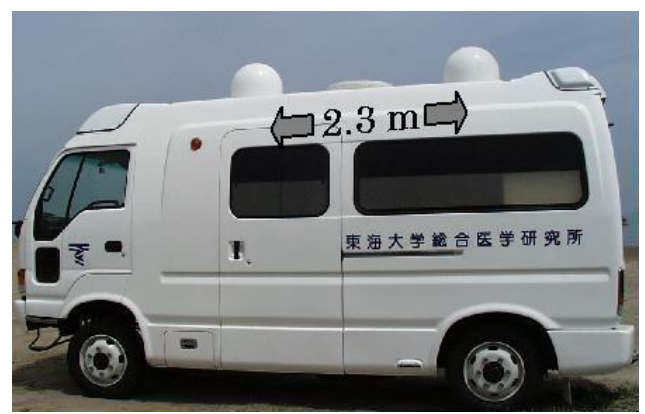

Fig. 18. Diversity_distance of $2.3 \mathrm{~m}$ for the prototype of the nextgeneration ambulance.

\subsection{Is it stable at an inclination of $\mathbf{4 0}$ degrees?}

Although Russia's MOLNIYA orbit (12 hours) or TUNDRA orbit ( 24 hours) have provided practical service for years in high-latitude areas, the inclination angles involved are almost 63.4 degrees. The Earth is not a perfect sphere; its oblate shape generates HEO anomalies. The formula for these fluctuations is called $\mathrm{J} 2$; assuming a minimum, we choose an angle of inclination of 63.4 degrees (Figures 19 and 20).

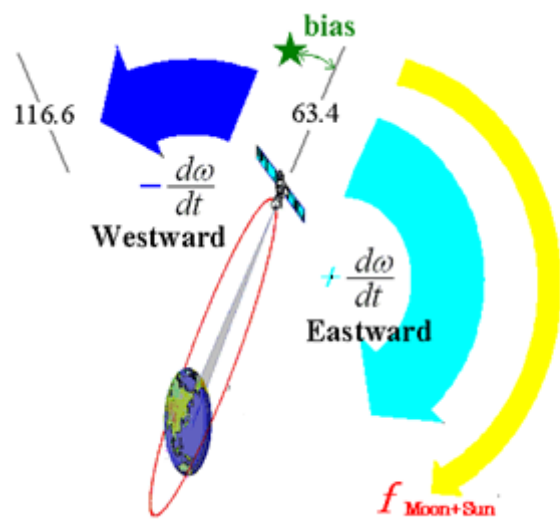

Fig. 19. External force applied to HEOs.

\section{Third body}

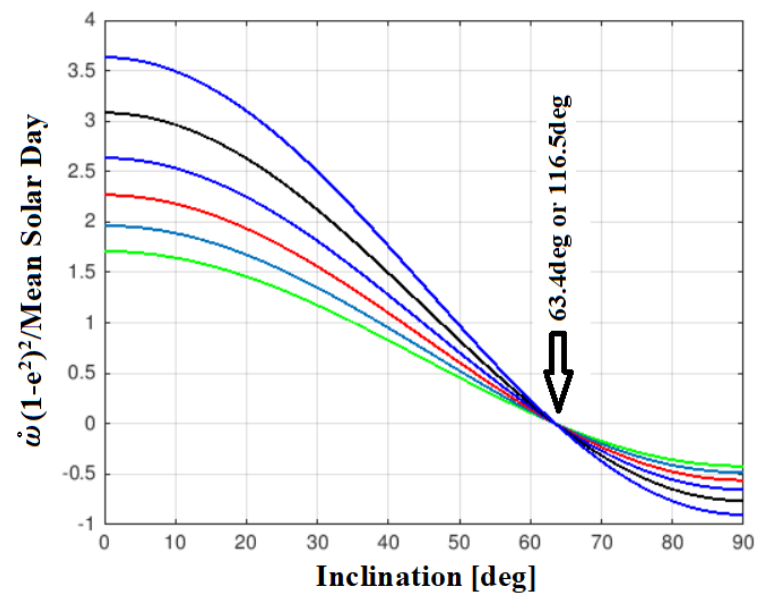

Fig. 20. Apsidal rotation rate due to oblateness vs. inclination for values of average altitude. 63.4 degrees is the ideal inclination for HEOs.

The TUNDRA orbit features significant eccentricity and an extended ellipse. Since the perigee is lower, the Tundra orbit must cross the Van Allen belt twice, resulting in degradations of solar array panels. Thus, we seek a lower eccentricity allowing passage over the Van Allen belt even at perigee. Figure 21 shows the orbital simulation calculated with MATLAB Oribitus ED. In this case, the height of the perigee is $23,137 \mathrm{~km}$, apogee $42,164 \mathrm{~km}$, inclination 40 degrees, and eccentricity 0.3 . This orbit would serve all of Japan. The J2 factor of external force from the oblateness is improved (1/1,000 times smaller) than the original TUNDRA orbiter (inclination of 63.4 degrees). The calculated service duration over the angle of elevation 70 degree would cover Wakkanai for 450 minutes and Tokyo, Nagoya, and Osaka for $540 \mathrm{~min}$./one(1) HEO (Figure 22). The minimum elevation angle with four (4) HEOs system shown in Figure 23. For Wakkanai, the figure improves from 37 with N-STAR GEO to 75 degrees. 

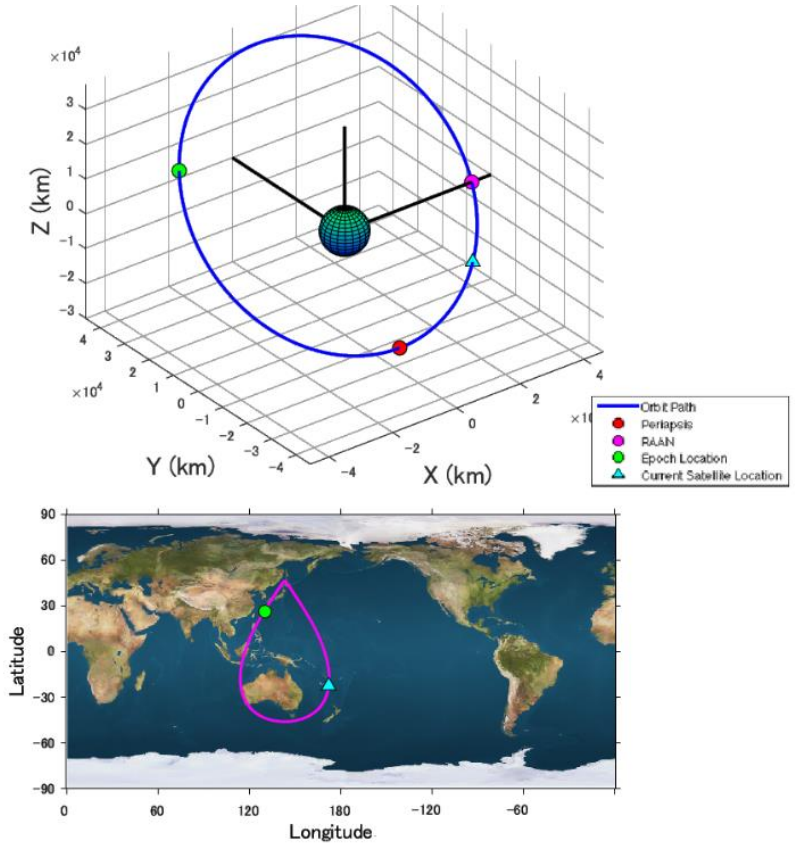

Fig. 21. Expected HEOs for Ambulatory applications.

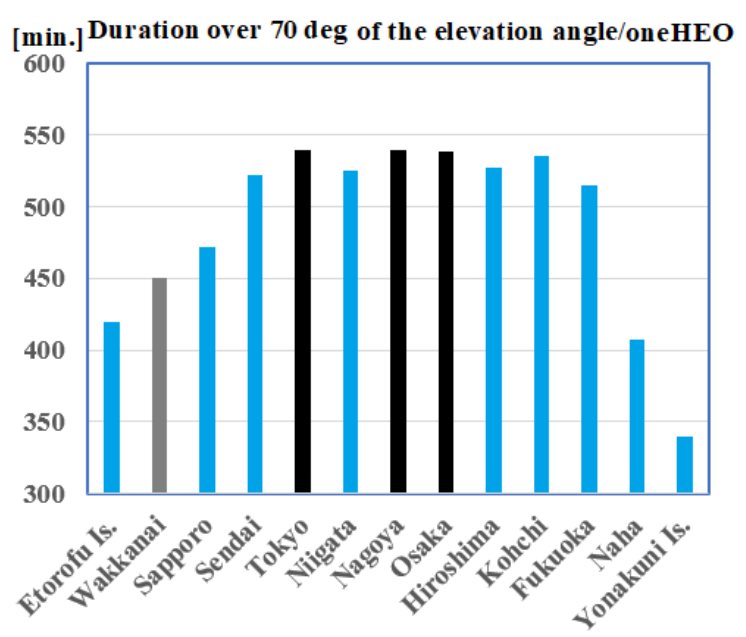

Fig. 22. Service duration over visibility angle 70 degree

Assuming four HEOs launched with Constellation shifted 6 hours, an angle of elevation by the average value of 24 hours of Tokyo, Nagoya, and Osaka will exceed 80 degrees.

\subsection{Combination operation with HEOs and 5 G/Ka- band}

Concerning about forward linkage of video transmission from moving vehicles to a center, this operation condition must be connected a broadband circuit continuously such as 5G/Ka-band and/or HEOs. 5G/Ka-band uses $28 \mathrm{GHz}$. This means the frequency characteristics related to blockage and shadowing are dramatically different from the lower microwave frequencies. Communication distances for $5 \mathrm{G} / \mathrm{Ka}-$ band will be $100-200 \mathrm{~m}$. Since the operating principle is primarily line-of-sight, carriers will need to deploy many repeaters and transponders across the urban landscape. In case of $5 \mathrm{G} / \mathrm{Ka}$-band, it is predicted that short break in the frame level occurs through repeaters and transponders. We are expecting combination operations with HEOs and $5 \mathrm{G} / \mathrm{Ka}$-band to solve this problem, because HEOs can keep line-of-sight propagation with high elevation angle for long duration. In such operations, the communications profile on the vehicle based on actual optical data will be very useful to predict blockages and to select/switch a suitable circuit.

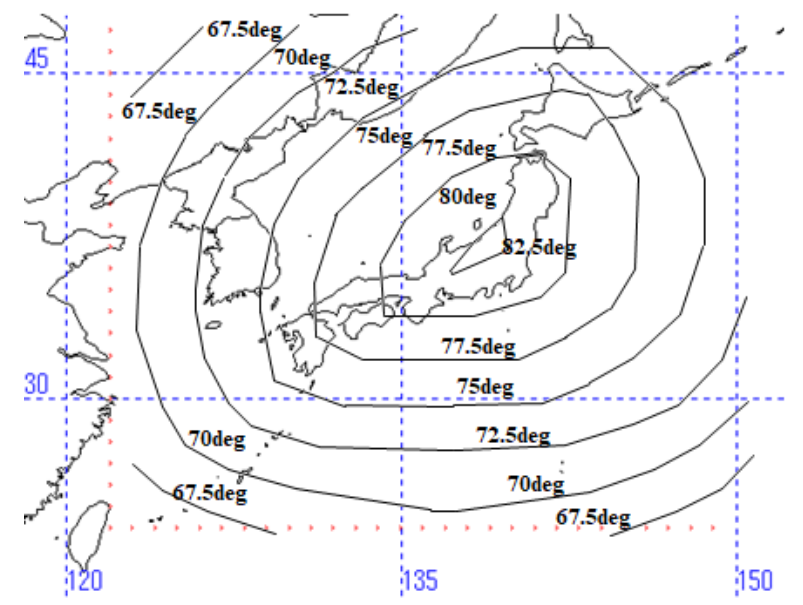

Fig. 23. Minimum elevation angle with four (4) HEOs system designed by Dr. Toshihide Maeda, Hitachi Co. Ltd.

\section{CONCLUSIONS}

Visibility to a satellite is easily determined by checking the value of the pixels in the binarized fisheye image of its position. The process of determining visible satellites is performed automatically. Although surely there is a difference from an optical course and microwave path by multipass (reflection) and/or Loo distribution, our field results are nearly identical to those given by Karasawa's formula. The launch and introduction of HEOs would significantly alleviate issues related to obstructions in urban areas in the north of Japan (e.g., Wakkanai) and densely urbanized areas like Ginza in Tokyo.

Here we suggest that HEOs could replace $5 \mathrm{G}$ in rural areas where $5 \mathrm{G}$ is unavailable. In addition, we show that mobile communication profiles based on actual optical data collection can be useful in predicting line-of-sight propagation and/or signal losses for $5 \mathrm{G}$ service.

\section{Acknowledgement}

This field research was fully supported by the Japanese Science and Technology Agency Special Coordination Funds for Promoting (Research and development for applying advanced satellite communication technology to medical care, Principal Investigator: Prof. Kiyoshi 
Kurokawa), and supported as the NEDO-subsidized project (Optical Analysis and Data Creation for Urban Environments, Principal Investigator: Isao Nakajima). We wish to thank the timely help given by Prof. Kiyoshi Kurokawa, the National Graduate Institute for Policy Studies, Dr. Toshihide Maeda, Hitachi Co. Ltd., Prof. Toshihiko Kitano, Dr. Nadia El Borai Yamamura, Dr. Futoshi Ohyama, Ms. Noriko Numata, Ms. Kimiko Kobayashi, and Ms. Miyoshi Tanaka in analyzing the large number of field data.

\section{REFERENCES}

[1] L. Husson, J.C. Dany, S. Chambon, K. Berradi and A. Beffani, "Modeling of mobile satellite channels by scalable clustering algorithm", Vehicular Technology Conference 2002. VTC Spring 2002. IEEE 55th, vol. 4, pp. 2004-2007, 2002.

[2] I. Dey, G. G. Messier and S. Magierowski, "The Cumulative Distribution Function for the Joint Fading and Two Path Shadowing Channel: Expression and Application", Vehicular Technology Conference (VTC Fall) 2014 IEEE 80th, pp. 1-5, 2014.

[3] M. Dottling, A. Jahn, D. Didascalou and W. Wiesbeck, "Two- and three-dimensional ray tracing applied to the land mobile satellite (LMS) propagation channel", Antennas and Propagation Magazine IEEE, vol. 43, no. 6, pp. 27-37, 2001.

[4] A.Subekti, H. Juzoji and I. Nakajima, "Technique for optical measurement of the urban environment, Mobile Communications Technology for Medical Care and Triage" MCMT 2002, published by APT-HRD (ISBN:979-9299-85-3), 2002.

[5] A.Subekti, H. Juzoji and I. Nakajima, "Technique for optical measurement of urban environment" Proceedings of AIAA-ICSSC Yokohama,pp.1-6, 2003.

[6] Y. Karasawa, K. Kimura and K. Minamisono, "Analysis of availability improvement in LMSS by means of satellite diversity based on three-state propagation channel model" IEEE Transactions on Vehicler Technology vol. 46, no.4, pp.1044-1056, 1997

[7] T. Kitano, H. Juzoji, I. Nakajima, "Elevation Angle of QuasiZenith Satellite to Exceed Limit of Satellite Visibility of Space Diversity which consisted of Two Geostationary Satellites", IEEE Transactions on Aerospace and Electronic Systems, vol. 48, no. 2, 2012, pp. 1779-1785.

[8] R.Khan and K. Kiasaleh, "Experimental validation of LMSS fading models for GPS measurements in fading/shadowing environment", Radio and Wireless Symposium (RWS) IEEE 2011, pp 8-12.2011, 2011.
[9] L. Chapman, J. E. Thornes and A. V. Bradley, "Sky-view factor approximation using GPS receivers", International Journal of Climatology, vol.22, pp.615621, 2002.

[10] J. S. Sanchez, A. Grlerhmann, P. Thevenon, P. Brocard, A. B. Afia and O. Julien, "Use of a fisheye camera for GNSS NLOS exclusion and characterization in urban environments",HAL.https;//hal-enac.archivesouvertes.fr/hal-01271970. 29 Nov. 2017.

[11] L.E. Braten, T. Tjelta, "Semi-Markov multistate modeling of the land mobile propagation channel for geostationary satellites", Antennas and Propagation IEEE Transactions on, vol. 50, no. 12, pp. 1795-1802, 2002.

[12] D. R. Iglesias, M. G. Sanchez, "Semi-Markov Model for Low-Elevation Satellite-Earth Radio Propagation Channel", Antennas and Propagation IEEE Transactions on, vol. 60, no. 5, pp. 2481-2490, 2012.

[13] R. Akturan, W. J. Vogel, "Photogrammetric mobile satellite service prediction" N95-14679, NASA https://ntrs.nasa.gov/

[14] S. Tongleamnak and M. Nagai,"Simulation of GNSS availability in urban environments using a panoramic image dataset" International Journal of Navigation and Observation vol.2017, pp.1-12, 2017.

[15].A. Nakajima, et. al. "Concept Study on Small Satellite for Mobile Communication", Conference Proceedings Vol. 3 WPMC'01 pp1223-1228, published by CRL, YRP and SCAT (ISBN: 87-988568-0-4), 2001.

[16] I. Nakajima, et. al."Cost Comparison between HEO Satellite and 3G Wireless Mobile Phones for Video Transmission from Ambulance", Conference Proceedings Vol. 3 WPMC'01 pp1573-1578, published by CRL, YRP and SCAT (ISBN: 87-988568-0-4), 2001.

[17] I. Nakajima, et. al. "Highly Inclined Elliptical Orbiter for Motion Picture Transmission from Ambulance, Conference Proceedings vol. 3 WPMC'01 pp1585-1590, published by CRL, YRP and SCAT (ISBN: 87-988568$0-4)$

[18]. R. Akturan, "An overview of the Sirius satellite radio system," International Journal of Satellite Communications and Networking, Special Issue: Special issue on Mobile Satellite Radio, vol.26, no.5, pp. 349-358, 2008

[19] St. D. Ilcev, "Highly elliptical orbits (HEO) for high latitudes and polar coverage" IEEE 20th International Crimean Conference "Microwave \& Telecommunication Technology, 2010

[20] Propagation data required for the design of Earth-space land mobile telecommunication systems, Recommendation ITU-R P.681-6, 2003 
[21] Maximum permissible level of off-axis r.i.r.p. density from very small aperture terminals (VSAT), Recommendation ITU-R S.728-1, 1995.

\section{Authors}

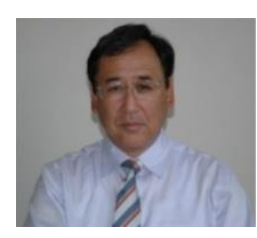

Isao Nakajima currently serves as Professor at the Nakajima Laboratory, Department of Emergency and Critical Care Medicine, Tokai University School of Medicine. Obtained an MD from the Tokai University School of Medicine in 1980; a PhD from the Graduate School of Advanced Medical Science, Tokai University, in 1988; and a PhD in Applied Informatics from the Graduate School of Applied Informatics, University of Hyogo, in 2009. A research goal for several years has been to identify ways to send vast volumes of multimedia data from moving ambulances via communications satellite to assist patients in critical condition.

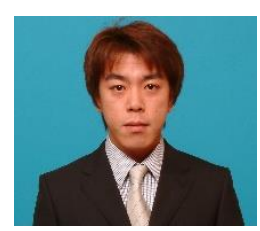

Kazuhide Tsuda graduated from Josai University in 1999; served from 2001 to 2003 as research fellow at the Nakajima Laboratory, Tokai University Institute of Medical Science._In addition to work on developing this experimental technology for deployment in the field, he has worked to present and promote understanding of the system through meetings and conferences all over the world, including events organized by APT, WSIS, and ITU Telecom World. Currently manages a trading company for technical products and machinery.

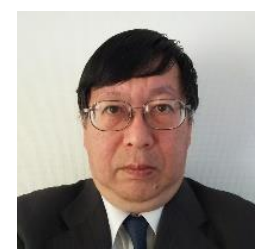

Hiroshi Juzoji graduated from the Tokai University School of Medicine in 1986; has studied issues related to telemedicine and eHealth for years, developing circuit designs and firmware for special equipment for experimental and practical use. In 1992, visited and installed small satellite earth stations in the AsiaPacific region for the Asia Pacific Medical Network via ETS-V. Developed the embedded tracking system and software with MATLAB for optical analysis for the work presented in this paper.

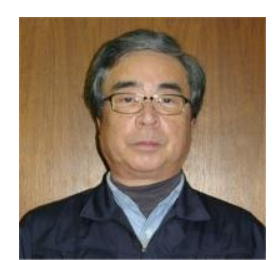

Masuhisa Ta graduated from the College of Engineering, Ibaraki University in 1975. Helped develop_ETS-V satellite mobile earth stations for the Asia Pacific Medical Network in 1990 and the Ku-band quadrant detector used to track the GEO (N-STAR) satellite from moving ambulances in urban areas. Granted wireless license for these transceiver units. Currently CEO of Tasada Works, with research interests in general machinery and the equipment manufacturing industry.

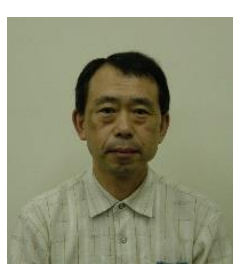

Atsushi Nakajima graduated from Shinshu University of Electrical Engineering and finished his Master Course in 1972. Awarded a $\mathrm{PhD}$ Doctor of Engineering from Kyoto University in 1983. Active from 1972 to 2008 in research and development on small satellite technologies at JAXA (Japan Aerospace Exploration Agency). Currently director of the Nakajima Space Engineering Laboratory for consulting on space technologies. 
A Nationwide Study on Optical Analysis for Expecting HEOs to Support Ambulances 